\title{
Shear-Wave Elastography in diagnostics of primary hyperparathyroidism - a new application of the method.
}

Adam Stangierski 1, Kosma Woliński 1, Ewelina-Szczepanek-Parulska 1, Edyta Gurgul 1, Maciej Biczysko2, Agata Czarnywojtek 1, Marek Ruchała

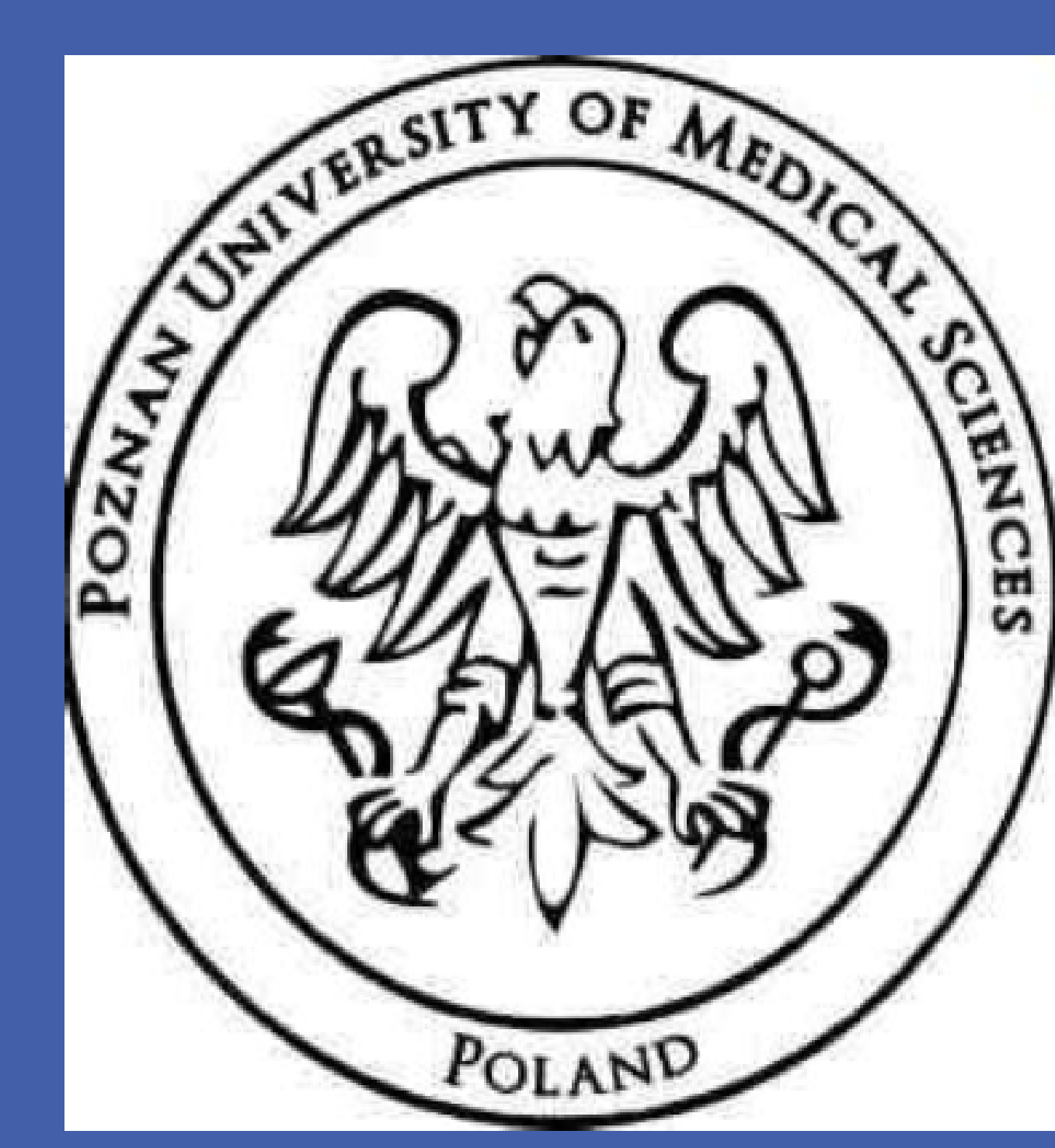

\section{Introduction}

Shear Wave Elastography (SWE) is a reliable, objective and reproducible technique in sonographic assessment of tissue stiffness. It is considered to be an improvement of conventional ultrasonographic examination. It was demonstrated to be useful in the diagnosis of many thyroid disorders, such as thyroid cancer, Hashimoto's thyroiditis or Grave's disease. The aim of our study was to check if SWE can be supportive in the diagnosis of primary hyperparathyroidism.

\section{Study group, methods}

43 patients referred for the surgery due to primary hyperparathyroidism were included. In all cases presence of parathyroid adenoma was confirmed by histopathology. Control group consisted of 322 benign thyroid nodules in 98 patients referred for surgery. Maximal elasticity (Emax) of each lesion was recorded.

\section{Results}

Mean Emax value for parathyroid adenomas was $14.4 \mathrm{kPa}$ with standard deviation (SD) $17.1 \mathrm{kPa}$; median was $11.1 \mathrm{kPa}$. For benign thyroid lesions mean Emax was equal to 57.3 with $\mathrm{SD}=60.6$, median $-36.2 \mathrm{kPa}$. The difference was statistically significant $(<0.05)$. Emax of the parathyroid adenomas was inversely correlated with PTH level $(\mathrm{p}=0.04, \mathrm{r}=-0.32)$.

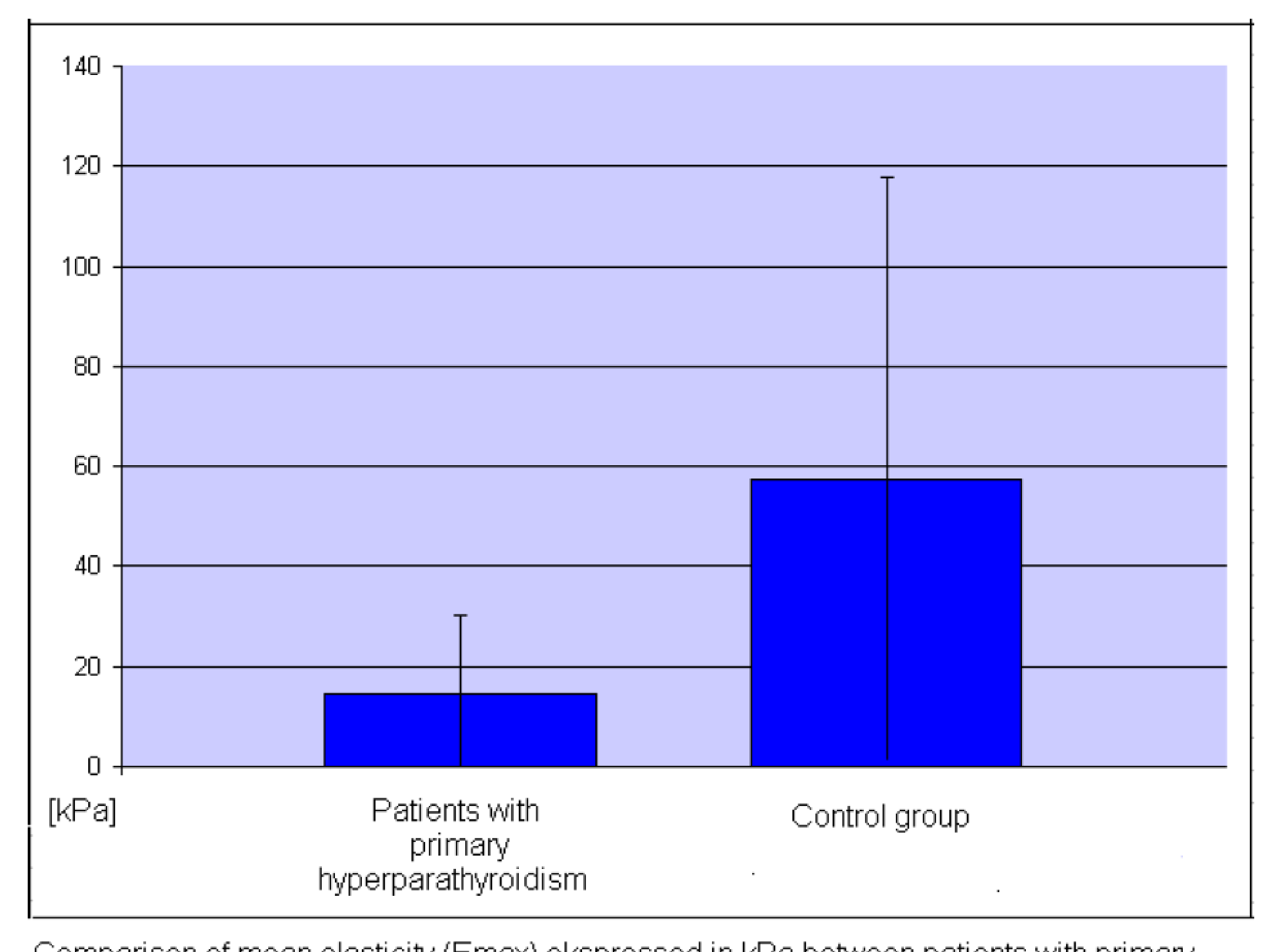
Comparison of mean elasticity (Emax) ekspressed in $\mathrm{kPa}$ between patients with primary
hyperparythyroidism and control group - benign thyroid lesions (meant standard deviation)
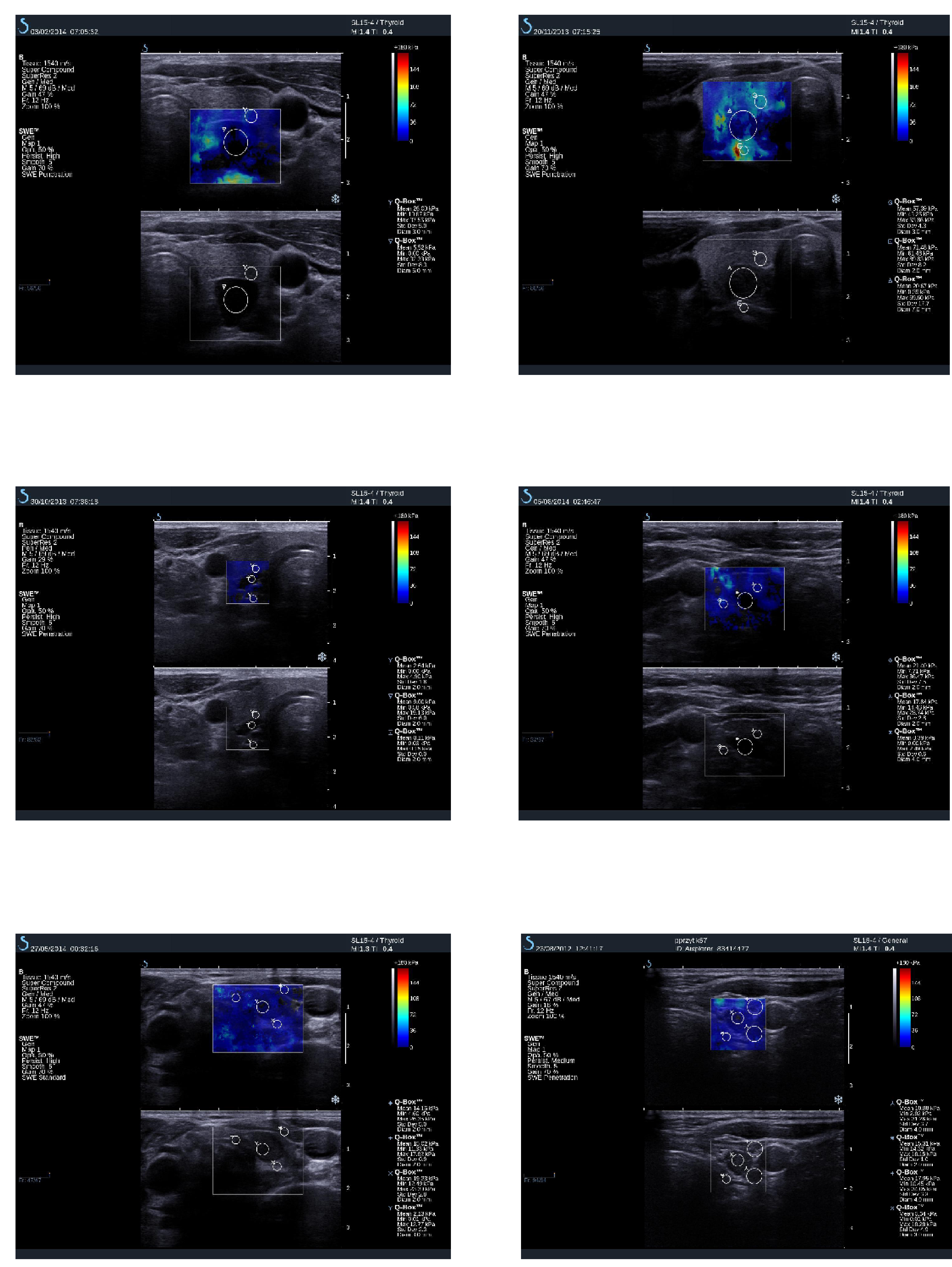

Pic.1-6. Shear wave elastography imaging in the assessment of parathyroid adenomas in some of the patients included in the study.

\section{Conclusions}

Parathyroid adenomas turned out to be significantly and distinctly more elastic than benign thyroid lesions - median values of the stiffness differed over three times. SWE can be a useful supportive method in the diagnosis of such lesions. Presence of very soft lesions, localized typically near the rear wall of the thyroid may arouse suspicion of primary hyperparathyroidism and hinting further diagnostic, such as measurement of $\mathrm{PTH}$, calcium and phosphate serum concentrations 